\title{
Chronic Pain Management in ENT Disorders
}

\author{
Anjana Sahu
}

\begin{abstract}
Chronic pain of otorhinolaryngology is considered to be the most disturbing and annoying condition. Suffering from chronic pain renders patient to loose his interest and ability to do the work. It may be responsible for many days lost from workplace, which affect directly or indirectly his quality of life. Patient may suffer from emotional and mental disturbances along with physical pain. Trigeminal neuralgia, cluster headache, otalgia, sphenopalatine and glossopharyngeal neuralgia and cancer pain are the some examples of chronic pain related to otorhinolaryngology. Multidisciplinary approach to treat the chronic pain helps the patients to get rid of their suffering and maintain their normal lifestyle.
\end{abstract}

Keywords: Chronic pain, Glossopharyngeal block, Sphenopalatine block, Trigeminal block.

How to cite this article: Sahu A. Chronic Pain Management in ENT Disorders. Int J Otorhinolaryngol Clin 2015;7(1):35-39.

\section{Source of support: Nil}

Conflict of interest: None

\section{INTRODUCTION}

Chronic pain is defined as any pain lasting more than the period of 3 months. It is frequently associated with fatigue, decreased appetite, sleep disturbances, mood swings and irritability. If left untreated may lead to disability and despair. Therefore, treating a patient with chronic pain requires both counseling and pharmacological treatment as it affects both the body and the mind.

\section{CAUSES OF CHRONIC PAIN IN ENT}

\section{Headache}

Sinusitis headache: Chronic sinusitis rarely causes chronic pain.

Cluster headache: It is usually unilateral pain in frontal, ocular and temporal areas, but sometime experienced in

\section{Assistant Professor}

Department of Anesthesiology, Topiwala National Medical College \& BYL Nair Charitable Hospital, Mumbai, Maharashtra India

Corresponding Author: Anjana Sahu, Assistant Professor Department of Anesthesiology, Topiwala National Medical College \& BYL Nair Charitable Hospital, Mumbai-400008, Maharashtra, India, Phone: 09969645713, e-mail: drdksahujrh@ gmail.com infraorbital and maxillary region. Attack of pain grouped in bouts, lasts for several weeks to months, with pain-free intervals of several months. The pain is excruciating. It is described as constant, stabbing, burning and throbbing. Pain is associated with ipsilateral ptosis, miosis, tearing, rhinorrhea and blocked nose. Chronic cluster headache is similar to cluster headache, but is rare presentation. Lithium carbonate works better in chronic cluster headache.

\section{Otalgia}

Intrinsic causes: Malignant otitis externa, infected cyst of ear canal, carcinoma of the ear, etc. ${ }^{1}$

Extrinsic cause: Referred otalgia arises due to involvement of cranial nerve either due to inflammation or due to compression.

Cranial nerve $V$ (trigeminal): Trigeminal neuralgia rarely results into otalgia but carcinoma of oral cavity can cause referred ear pain.

Cranial nerve VII (facial): Dental problems, temporomandibular joint (TMJ) syndrome or parotid gland disorders.

Cranial nerve IX (glossopharyngeal): Pharyngitis or tonsillitis, carcinoma of oropharynx.

Cranial nerve X (vagus): Carcinoma of laryngopharynx, or from esophagus in gastroesophageal reflux disease (GERD).

Spinal nerve C2 and C3: Discomfort over mastoid due to involvement of greater auricular nerve and lesser occipital nerve. Cervical spine disk disease or cervical meningioma may cause referred ear pain.

\section{Neck Pain}

Thyroiditis, mumps, greater occipital neuralgia, myofascial pain, cervical spondylitis and traumatic torticollis are some main causes of chronic pain in neck.

\section{Evaluating Pain}

- History of long-standing pain along with chronic health issues, irritability, anxiety, depression, psychiatric ailments and poor performance are suggestive of chronic pain disorder.

- Mode of onset, location, nature, intensity, frequency, time, duration of pain should be asked. Aggravating and relieving factors may give us important clues while making diagnosis. Primary neuralgias are described as sharp and lancinating, myofascial pain 
as continuous dull aching and vascular headache as throbbing. Aggravating factors (lying down, stress, the sight and smell of food, chewing, alcohol) and relieving factors (heat and cold) are important questions should be asked during evaluation of pain. Presence or absence of associated factors, such as flushing, tearing, swelling and nasal congestion should be ascertained. Trigeminal neuralgia is often described as paroxysmal, jabbing or shock-like pain in contrast to non-neuropathic pain, such as TMJ dysfunction, which is a unilateral, dull, aching pain around periauricular region.

- Past history of pain management or other medication should be asked.

\section{Assessment and Diagnosis}

- Various single dimension scales are available to assess the pain.

Visual analog scale (VAS): The VAS is a most common scale to assess pain. It is a straight $100 \mathrm{~mm}$ line, has words 'no pain' at the left most end and 'worst pain imaginable' at the right most end. Patient is asked to keep his finger on this straight line, indicating amount of pain that he feels.

Numerical rating scale (NRS): It is similar to VAS but with marked number from 0 to 10 on straight line. Zero means no pain and 10 means worst pain.

Verbal descriptor scale (VDS): This scale comprises of certain words, such as none, mild pain, moderate pain, severe pain or no pain, mild, discomforting, distressing, horrible and excruciating pain.

- Multidimensional scales mainly useful in assessing chronic pain.

McGill pain questionnaire (MPQ): McGill pain questionnaire is a type of questionnaire to be asked at the time of evaluation. It is reliable, valid and consistent in its ability to assign appropriate description of pain. Short-form McGill pain questionnaire (SF-MPQ), brief pain inventory (BPI), memorial pain assessment card (MPAC), multidimensional affect and pain survey (MAPS)

- Complete general examination along with routine blood and urine analysis. Also radiograph and other required imaging techniques, such as computed tomography or magnetic resonance imaging (MRI) should be carried out to confirm the clinical diagnosis.

\section{Management}

Multidisciplinary approach should be the principle of treatment of chronic pain disorder. It involves the teamwork of pain physician, anesthesiologist, radiologist and psychiatrist.
Psychotherapy: Presence of depression and anxiety in chronic pain requires psychotherapy as an important component of successful management. ${ }^{2}$

Pharmacologic therapy: Chronic pain management requires more than one drug therapy for treating a condition. $^{2}$

- Anticonvulsants have become the first line treatment of neuropathic pain syndrome. ${ }^{3,4}$ Old generation anticonvulsants, such as carbamazepine, phenytoin, valproate and clonazepam have been used for treating neuropathic pain. Now new generation anticonvulsants, such as pregabalin, topiramate, oxcarbazepine, lamotrigine, zonisamide and levetiracetam are available to treat chronic pain. Generally only one anticonvulsant should be prescribed at a time and upward titration should be based on efficacy and tolerability.

- Antidepressant, such as tricyclic antidepressant especially amitriptyline has been used since many years. Selective serotonin reuptake inhibitor, serotoninnorepinephrine reuptake inhibitor and dopaminergicmediated antidepressant are efficacious in neuropathic pain.

- Narcotic analgesics are most potent prescription analgesic. Start with short acting narcotic and gradually switch to a long acting if required depending on patient's need. Tramadol hydrochloride is a unique narcotic medication and its efficacy in treating chronic pain put it into as first line drug before a more traditional narcotic analgesic prescribed. ${ }^{5}$

\section{Interventional Strategies}

Nerve blocks may be helpful for diagnostic purposes as well as therapeutic in breaking the chronic pain cycle.

Sympathetic blockade has also been used for both diagnostic and therapeutic purposes.

\section{Physical Therapy}

Transcutaneous electric nerve stimulation unit.

\section{Complimentary Strategies}

Acupuncture for treating myofascial pain.

\section{Massage Therapy}

Nutritional counseling.

\section{Special Consideration}

\section{Trigeminal Neuralgia}

Trigeminal neuralgia is a severe unilateral (usually) facial pain, characterized by lancinating pain in the distribution of one or more division of trigeminal nerve. 
Annual incidence is of 4 to 5 per 100,000 , and most frequently seen in elderly population. ${ }^{6}$ Light touch in the distribution of trigeminal territories specially $V_{2}$ and $V_{3}$ results into onset of paroxysm of pain. Other triggering factors are chewing, talking, brushing teeth, cold air, or smiling or grimacing, etc. may play a role in the onset of pain. Some patients may experience pre-trigeminal neuralgia in the form of dull, continuous, aching pain in jaw, which eventually turn up into trigeminal neuralgia. Pathophysiology of trigeminal still not fully understood but it is said that demyelinating lesion of trigeminal nerve set up ectopic impulses. Magnetic resonance imaging and magnetic resonance angiography should be performed to look for demyelinating lesion, a mass lesion in cerebellopontine angle or an ectatic blood vessel. Trigeminal neuralgia is usually successfully treated with anticonvulsant, gabapentin, lamotrigine, neuroleptic drugs as described earlier in management section and misoprostol, a prostaglandin $\mathrm{E}$ analog in trigeminal neuralgia caused by multiple sclerosis. Patient refractory to pharmacological therapy needs surgical interventions. Various surgical procedures available, one of them is microvascular decompression described by Janetta, ${ }^{7}$ but it requires intracranial approach with a small morbidity and mortality rate. Direct targeting trigeminal nerve includes percutaneous radiofrequency rhizotomy, glycerol rhizolysis, and balloon compression. Recently gamma knife therapy shows excellent results. Patient who is not tolerating aggressive therapy may be benefitted with peripheral nerve root avulsion.

\section{Cluster-tic Syndrome}

Coexistence of cluster headache and trigeminal neuralgia simultaneously is known as cluster-tic syndrome. ${ }^{8}$ There are three types of pain: first component includes trigeminal type of pain paroxysmal, extremely brief, and severe. Second component includes cluster headache type pain with autonomic phenomena (lacrimation, rhinorrhea). Third component has mixture of the first two. Medical management usually unsuccessful although surgery relieves neuralgia, and cluster like pain may be lessened and responsive to medical treatment.

\section{Glossopharyngeal Neuralgia}

Glossopharyngeal neuralgia is described as occurrence of paroxysmal pain in the distribution of cranial nerve IX and X. Severe unilateral paroxysmal pain affecting ear, larynx, tonsil, or tongue. Triggering factors includes chewing, talking, swallowing, coughing, yawning, certain tastes and touching the neck or external auditory canal. Medical treatment is of same as trigeminal neuralgia. Application of local anesthetic to oropharynx is helpful in diagnostic as well as therapeutic purposes. Injection of local anesthetics into the stylohyoid ligament can be diagnostic if Eagle's syndrome (in which cranial nerve IX is compressed laterally against an ossified stylohyoid ligament) is strongly considered. Surgical treatment includes intracranial sectioning of cranial nerve IX at jugular foramen or vascular decompression.

\section{Sphenopalatine Neuralgia}

It is described as unilateral, episodic, perinasal facial pain with nasal congestion. Treatment includes pharmacological therapy, intranasal transmucosal application of local anesthetic and fluoroscopic guided sphenopalatine ganglion block.

\section{Cancer Pain Management Modalities ${ }^{9}$}

\begin{tabular}{|c|c|}
\hline \multirow[t]{9}{*}{ Sytemic analgesic therapy } & Non-opioid analgesics \\
\hline & Opioid analgesics \\
\hline & Adjuvant \\
\hline & Antidepressant \\
\hline & Anticonvulsant \\
\hline & Local anesthetics \\
\hline & Corticosteroids \\
\hline & Other \\
\hline & Side effect management \\
\hline \multirow[t]{4}{*}{ Invasive therapy } & Peripheral nerve blocks \\
\hline & Plexus blocks \\
\hline & Neuraxial therapy \\
\hline & Neuroablative therapy \\
\hline \multicolumn{2}{|l|}{ Antitumor therapy } \\
\hline \multicolumn{2}{|l|}{ Psychosocial therapy } \\
\hline \multirow{8}{*}{ Nonpharmacologic therapy } & Heat and cold application \\
\hline & Exercise \\
\hline & Counterstimulation (TENS) \\
\hline & Relaxation/imagery \\
\hline & Distraction/reframing \\
\hline & Hypnosis \\
\hline & Occupational therapy \\
\hline & Physical therapy \\
\hline
\end{tabular}

\section{Blocks for Chronic Pain Management in ENT Gasserian Ganglion Block}

Trigeminal nerve is the largest and most complex cranial nerve, containing sensory and motor fibers. Gasserian ganglion is formed from two roots that exit the ventral surface of the brainstem at the midpoint level. It is canoeshaped and has three sensory division, the ophthalmic (V1), the maxillary (V2), and mandibular (V3) nerves, which exit on the anterior convex aspect. A small motor root joins the mandibular nerve and exit through foramen ovale. 


\section{Indication}

Palliation of cancer pain

Trigeminal neuralgia

Cluster headache

Intractable ocular pain

\section{Technique}

The patient should be laid in supine with roll under the cervical spine, to extend it. Approximately $2.5 \mathrm{~cm}$ lateral to the corner of mouth, the area should be prepared with aseptic technique and anesthetized with local 1\% lidocaine. A $13 \mathrm{~cm} 20$ G Hinck needle is advanced, perpendicular to the pupil of eye (when eye is midposition) in a cephalad direction toward the auditory meatus. The needle is advanced until contact is made with base of the skull. The needle tip is withdrawn slightly and walked posteriorly into foramen ovale. Now remove the stylet of the needle and carefully aspirate for blood. Free flow of CSF may or may not be seen. The needle position should be confirmed by radiography before any local anesthetic or neuroleptic agent is injected. Once it is confirmed inject $0.1 \mathrm{ml}$ aliquots of preservative free local anesthetic, such as $1 \%$ lidocaine for diagnostic blocks and $0.5 \%$ bupivacaine for therapeutic blocks, or of sterile glycerol, $6.5 \%$ phenol in glycerine, or absolute alcohol may be injected. Average volume of $0.4 \mathrm{ml}$ of neurolytic agent is sufficient. This approach may be used for placing radiofrequency needles, cryoprobes and stimulating electrodes.

\section{Mandibular and Maxillary Nerve Block}

The patient should be laid supine and coronoid notch is palpated by asking the patient to open and close the mouth. After confirming the notch at the level of external auditory meatus ask the patient to hold his mouth in neutral position. A 3 and half inch $22 \mathrm{G}$ styletted spinal needle is inserted just beneath the zygomatic arch, at the midpoint of the coronoid notch. The needle is advanced approximately one and half to two inches perpendicular to the base of skull, until the lateral pterygoid plate is encountered. At this point both mandibular and maxillary block can be given. The needle is withdrawn approximately $1 \mathrm{~mm}$. After careful aspiration for blood, 7 to $10 \mathrm{ml}$ of preservative free local anesthetic is injected in small increments.

For selective maxillary nerve block, the styletted spinal needle is withdrawn and reinserted to skip just above the anterior margin of the lateral pterygoid plate. Maxillary paresthesia is observed, 3 to $5 \mathrm{ml}$ of preservative free local anesthetic is injected.

For selective mandibular nerve block, after localizing the lateral pterygoid plate, the styletted spinal needle is withdrawn a little and directed farther posteriorly and inferiorly. Mandibular paresthesia is observed, 3 to $5 \mathrm{ml}$ of preservative free local anesthetic is injected in small incremental doses.

For diagnostic block, $1 \%$ preservative free lidocaine is most suitable agent. For therapeutic blocks $0.5 \%$ bupivacaine in combination with $80 \mathrm{mg}$ of depot methylprednisolone (Depo-Medrol) is injected. If selective mandibular or maxillary nerve is desired, incremental $0.1 \mathrm{ml}$ of sterile glycerol, 6.5\% phenol in glycerine, or absolute alcohol may be injected. Total volume of $1 \mathrm{ml}$ of neurolytic agent is sufficient.

\section{Sphenopalatine Ganglion Block}

Sphenopalatine ganglion (SPG) is the largest collection of neurons outside the cranial cavity and second largest nerve center in head. It is situated in the pterygopalatine fossa just lateral to the lateral nasal wall at the level of the middle turbinate. This is a small pyramidal space, about $2 \mathrm{~cm}$ of height and $2 \mathrm{~cm}$ of width. It is bounded anteriorly with maxillary sinus and posteriorly by medial plate of pterygoid process.

\section{Indication}

- Atypical facial pain

- Migraine headache

- Cluster headache

- Sympathetically maintained pain of face

- Trigeminal neuralgia (typical tic douloureux and atypical).

- Sphenopalatine neuralgia.

\section{Technique}

Before going to perform this block a full anesthetic history must be obtained and keep ready resuscitation equipment in the vicinity. Any sign of tinnitus, perioral numbness and tingling, visual disturbances and dizziness are early signs of local anesthetic toxicity or intravascular injection.

\section{Transnasal Approach}

Two long cotton swabs soaked in the anesthetic solution are introduced into the naris of affected side. Advanced in perpendicular plane of face until a resistance is felt that is posterior of nasal cavity. Both the cotton swab should be introduced one after another and do not perform this block bilaterally in same sitting. While advancing swab, if patient complains of any discomfort, wait for few minutes and go ahead with the procedure. Now keep both the cotton swab in position for 35 to 45 minutes to allow the drug to cross nasal mucosal barrier and reach SPG. 


\section{Infrazygomatic Approach}

This approach is technically demanding and should be performed if transnasal approach is unsuccessful in reducing the pain. This is done under fluoroscopic guidance and with the use of specific curved blunt Coude' block needle. Coronoid process is palpated just anterior to this over mandibular notch, the area is prepared and anesthetized. Introduce the Coude' needle perpendicular toward pterygopalatine fossa under fluoroscopic guidance. Once reached negative aspiration done for blood, CSF, and air inject $2 \mathrm{ml}$ of local anesthetic solution and $1 \mathrm{ml}$ of $4 \mathrm{mg} / \mathrm{ml}$ dexamethasone. After the block inspect the area for any hematoma formation as one of the branch from facial artery runs near mandibular notch. Give constant pressure for 5 minute, it will be resolved.

\section{Radiofrequency Ablation}

This is performed in the same manner as described earlier, only difference is that the Coude' needle has $10 \mathrm{~mm}$ active tip RF needle sensory stimulator is performed at $50 \mathrm{~Hz}$ with a $20 \mathrm{~ms}$ pulse width and a voltage between 0.1 and $1.0 \mathrm{~V}$. Motor stimulation should then be performed using a $2 \mathrm{~Hz}$ cycle and voltage between 0.1 and $2.0 \mathrm{~V}$. Radiofrequency ablation (RFA) can be delivered at $80^{\circ} \mathrm{C}$ for 90 seconds.

\section{Glossopharyngeal Nerve Block}

Glossopharyngeal nerve contains both sensory and motor fibers. It exits the jugular foramen near the vagus and accessory nerves and internal jugular vein. One landmark for glossopharyngeal nerve is styloid process of temporal bone.

\section{Indication}

Prognostic nerve block before neurodestructive procedures:

- Neurolytic block and neurodestructive procedures

- Cancer pain (palliation)

- Management of glossopharyngeal neuralgia.

\section{Technique}

\section{Extraoral Approach}

Styloid process is located at a midpoint of imaginary line, drawn from mastoid process to angle of mandible. Patient lies supine and area prepared with aseptic precaution. A $22 \mathrm{G}, 1.5$ inch needle attached to a $10 \mathrm{ml}$ syringe is advanced at this midpoint in a plane perpendicular to the skin, till styloid process encountered at a depth of 2 to $3 \mathrm{~cm}$ after hitting the styloid process the needle should be withdrawn a little and walked off the process posteriorly. As soon as the bony contact is lost carefully inject $7 \mathrm{ml}$ of $0.5 \%$ preservative free lidocaine combined with $80 \mathrm{mg}$ methylprednisolone in incremental doses after negative aspiration of blood and CSF.

\section{Intraoral Approach}

A 22 G, 3.5 inch spinal needle that has been bent approximately $130^{\circ}$ is inserted through the mucosa at the lower lateral portion of posterior tonsillar pillar. The needle is advanced for $0.5 \mathrm{~cm}$. Inject the anesthetic mixture in the same manner as described in extraoral approach for glossopharyngeal nerve block.

\section{SUMMARY}

Orofacial or craniofacial pain is frequently encountered pain problem in ENT specialty. Chronic pain is usually neuropathic in origin pain with psychiatric ailment and it requires multidisciplinary approach for successful management.

\section{REFERENCES}

1. Keith DA. Orofacial pain. In: Warfield CA, Bajwa ZH, editors. Principles and practice of pain medicine. 2nd ed, India, New Delhi, Tata McGraw Hill; 2005:1. p. 252.

2. Hainline B. Chronic pain: physiology, diagnostic, and management considerations. In: Waldman SD, editor. Pain management. Philadelphia: Saunders Elsevier; 2007. p. 212.

3. Namaka M, Gramlich CR, Ruhlen D, et al. A treatment algorithm for neuropathic pain. Clin Ther 2004;26(7):951.

4. Tremont-Lukats IW, Megeff C, Backonja MM. Anticonvulsants for neuropathic pain syndromes: mechanism of action and place in therapy. Drugs 2000;60(5):1029.

5. Staats PS, argoff CE, Brewer R, et al. Neuropathic pain: incorporating new consensus guideline into the reality of clinical practice. Adv Stud Med 4 (7B): S550,2004.

6. Kateric S, Williams DB, Beard CM, et al. Epidemiology and clinical features of idiopathic trigeminal neuralgia and glossopharyngeal neuralgia: similarities and differences. Neuroepidemiology 1991:10(5-6):276-281.

7. Janetta PJ. Microsurgical management of trigeminal neuralgia. Arch Neurol 1985;42(8):800.

8. Ward TN, Levin M. Facial pain: In: Warfield CA, Bajwa ZH, editors. Principles and practice of pain medicine. 2nd ed. India, New Delhi, Tata McGraw Hill, 2005:1. p. 246.

9. Braiteh F, Bruera E. Palliative care in the management of cancer. In: Waldman SD, editor. Pain management. Philadelphia: Saunders Elsevier; 2007. p. 360. 